\title{
Case of arthritis secondary to leprosy
}

\author{
Fiaz Alam ${ }^{1 *}$ and Samar AL Emadi ${ }^{2,3}$
}

\begin{abstract}
Introduction: Leprosy is a chronic granulomatous infectious disease, which is caused by Mycobacterium leprae. High numbers of people are still affected by this disease in some of the developing countries however, it is rarely seen in non-endemic regions.

Cutaneous and neurological manifestations are the common and classical presentations of leprosy. Musculoskeletal involvement is the third most common manifestation but is less frequently reported.

Joint involvement can present as acute symmetrical polyarthritis or chronic polyarthritis resembling rheumatoid arthritis. Leprosy can also present with tenosynovitis, either isolated or associated with arthritis.

Case presentation: We report a case of 29-year-old man who developed tenosynovitis and acute symmetrical polyarthritis including small joints of hands and feet three weeks after appearance of typical cutaneous lesion of leprosy. Patient improved with steroid and anti leprosy treatment. Patient had another acute episode with symmetrical polyarthritis four months later while on treatment.
\end{abstract}

Discussion and Evaluation: In the modern era, there is increase movement of population from developing countries to developed countries, it is likely that patient suffering from leprosy with arthritis may present to rheumatology clinic in those countries where leprosy is not endemic. Exact pathogenesis for musculoskeletal manifestations is still not fully known. Our patient presented with symmetrical polyarthritis, enthesitis and systemic involvement, which was secondary to lepra reaction.

Having good knowledge of musculoskeletal manifestation of leprosy, will help narrow differential diagnosis and will prevent unnecessary diagnostic workup.

Conclusion: Leprosy can present with different rheumatologic manifestation including tenosynovitis and acute symmetrical polyarthritis. Type 2 lepra reaction (Erythema nodosum leprosum ENL) can present with systemic manifestation and can involve skin, nerves, joints, kidneys and liver.

\section{Introduction}

Leprosy is an infectious disease caused by Mycobacterium leprae. It is an important global health concern and needs early diagnosis and prompt treatment to prevent permanent disability.

Leprosy is multisystem granulomatous disease. It primarily involves the skin and peripheral nerves. The severity of neurological and cutaneous presentation depends upon the number of bacilli and type of immune response. It could be either pauci bacillary with intense immune reactions or multibacillary with weak immune response.

\section{Epidemiology}

115 countries submitted their reports to WHO (World health organization 2014) by the end of 2012. The global registered prevalence of leprosy at the end of 2012 was 189018 cases. The number of new cases reported globally in 2012 was 232857 compared with 226626 in 2011.

The global statistics (World health organization) show that 220810 (95\%) of new leprosy cases were reported from 16 countries and only $5 \%$ of new cases are from the rest of the world.

There are two different ways of classifying leprosy.

Ridley-Jopling (Pardillo et al. 2007) system and the simpler and more commonly used WHO classification system.

\section{Ridley-Jopling classification}

Leprosy is classified (Pardillo et al. 2007) into five different forms depending upon the immunological reaction and 
number of bacilli in skin lesions. Less number of bacilli in skin lesions and severe immunological reaction characterizes tuberculoid leprosy while lepromatous leprosy exhibits weak immunologic reaction and increase number of bacilli in skin lesions. These five different forms are Polar tuberculoid form, Borderline tuberculoid, Mid-borderline, Borderline lepromatous, and Polar lepromatous leprosy (Figure 1).

\section{WHO classification}

The WHO (Pardillo et al. 2007; WHO 2011) classifies leprosy according to the number of lesions and the presence of bacilli on a skin smear.

Paucibacillary (PB) leprosy is defined as five or fewer skin lesions. Bacilli are not seen on skin smears. Patients with only a single skin lesion are classified separately as single lesion PB leprosy. Multibacillary (MB) leprosy is defined as six or more lesions. In case of MB skin smear may be positive for bacilli.

If only number of skin lesion are considered then it can lead to overtreatment of paucibacillary leprosy as skin smear may be negative in patients with more then 5 skin lesion.

Musculoskeletal (Shejpal et al. 2014; Wakhlu and Agarwal 2010; Prasad et al. 2012; Atkin et al. 1990, 1987; Lopéz et al. 2012) manifestations are common in leprosy but less frequently reported. Joint involvement occurs in about $75 \%$ of cases of leprosy.

Different patterns of musculoskeletal presentation can occur in leprosy patient. Joint involvement can occur in form of monoarticular, oligoarticular or polyarticular. It can present as acute (Atkin et al. 1987) symmetrical polyarthritis or chronic symmetrical polyarthritis mimicking rheumatoid arthritis (Lopéz et al. 2012). Enthesitis (Atkin et al. 1990) and tenosynovitis are also seen in leprosy patient.

\section{Case presentation}

27 year old Nepali male presented to the emergency department with five days history of high grade fever, multiple joint pain and swelling preceded by four weeks history of progressive painful nodular skin lesion over the face, scalp, both ears, all four limbs and upper chest. Fever was not associated with headache, productive cough, abdominal pain, dysuria and diarrhea. Small joints of hands, bilateral elbows, knees and feet were painful. There was no history of preceding diarrhea, upper respiratory tract infection or urinary tract infection.

There were no previous similar complaints in the past.

On examination patient was febrile with temperature of 38 centigrade, blood pressure of $114 / 74$, pulse 95 per minute. His general appearance revealed multiple painful nodular skin lesions over face, both ears, upper and lower limbs. (See Figure 2). There were widespread hypo and hyper-pigmented skin lesions. Sensation over the skin lesion was intact.

Musculoskeletal examination revealed tender and swollen bilateral MCPS, PIPs, wrists, elbows, bilateral knees and bilateral $3^{\text {rd }}$ and $4^{\text {th }}$ MTPs. Patient also had evidence of enthesitis around both knees at site of quadriceps tendon insertion and tenosynovitis of wrist extensors bilaterally. Lung and cardiovascular examination was essentially normal. Neurological examination showed thickened ulnar nerves bilaterally with no evidence of ulnar neuropathy.

Cranial nerves were intact. Muscle power in all groups of muscles was normal. There was no sensory impairment at any level.

Laboratory investigations revealed WBC count of 36800 (normal 4-10). (93\% neutrophils), Hemoglobin $(\mathrm{Hb})$ of $8.7 \mathrm{~g} / \mathrm{dl}(\mathrm{MCV}$ of $74.6, \mathrm{MCH}$ of 22.3$)$, Platelet counts of $656 \mathrm{~K}$; blood urea nitrogen $13.6 \mathrm{mmol} / \mathrm{L}$ (normal 2.5 -6.7) and creatinine 163umol/L normal (50.4-98.1). Inflammatory markers were raised (CRP of $335 \mathrm{mg} / \mathrm{l}$
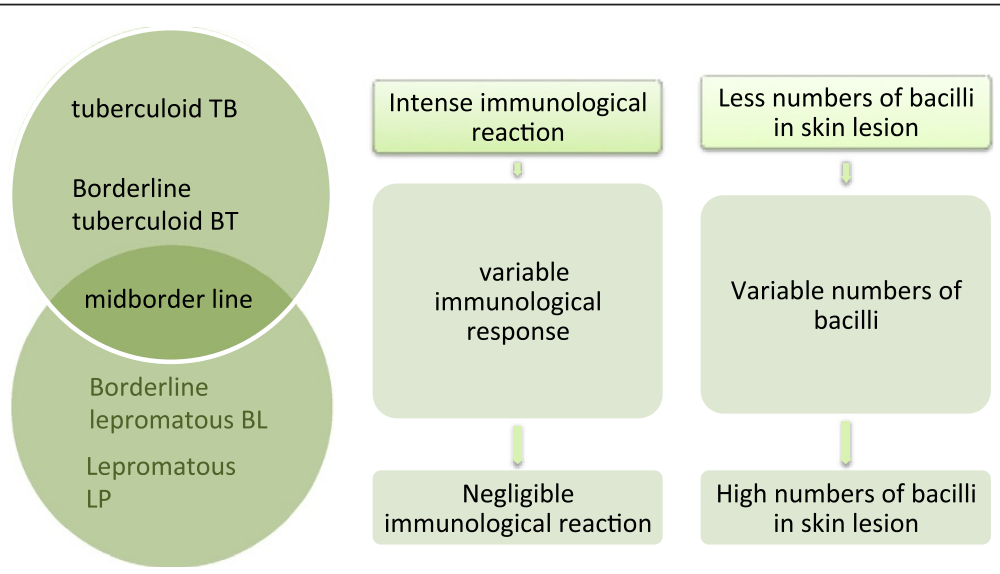

Figure 1 Ridley-Jopling classification. 


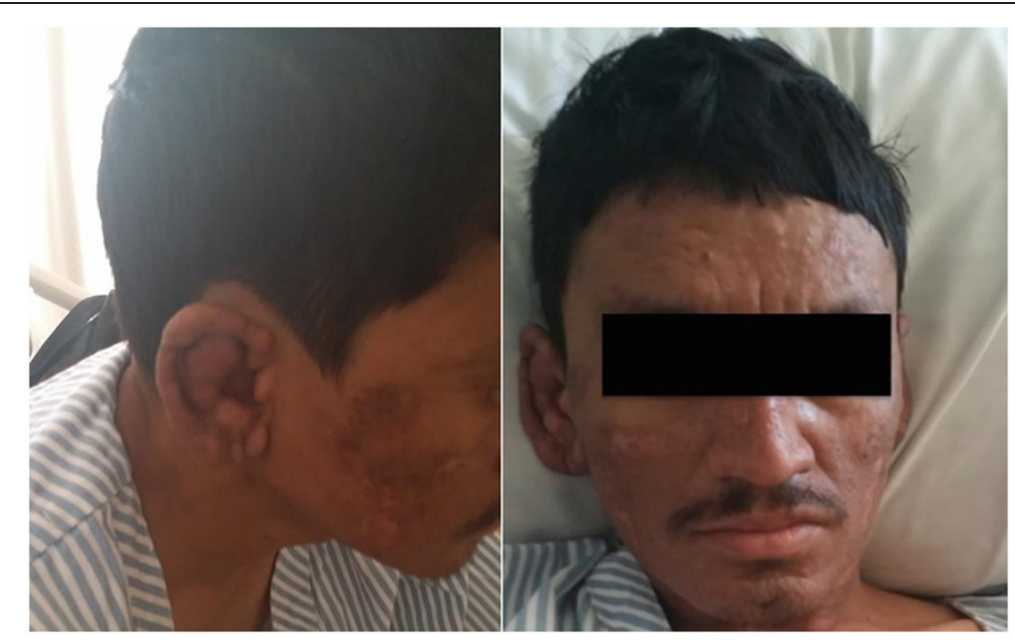

Figure 2 Papulo-nodular skin lesions over the face and ear.

and ESR of $87 \mathrm{~mm} / 1 \mathrm{hr}$ ). Urine chemical examination showed $2+$ protein and $3+$ erythrocytes.

Urine Microscopy revealed WBC count of 103 and Red blood cell of 486 with positive granular cast.

Urinary protein creatinine ratio was $117 \mathrm{mg} / \mathrm{mmol}$ (normal range of $0-45$ ).

Blood and urine culture didn't grow any organism.

Further investigation revealed negative ANA, RF, anti ccp and normal complements. $\mathrm{X}$ rays of hands and feet were normal.

Punch skin biopsy (see Figure 3 histopathology of skin nodule) was taken from the nodule present over right upper arm. Skin biopsy revealed non-necrotizing granuloma with numerous foamy macrophages in dermis and subcutaneous fat. Small number of acid-fast bacilli was seen in some of the macrophages and biopsy result was reported as borderline tuberculoid. Leprosy was diagnosed on basis of typical skin lesion and skin biopsy finding.

Treatment of our case included intravenous hydration and anti-leprosy medication (rifampicin $600 \mathrm{mg}$ monthly and dapsone $100 \mathrm{mg}$ daily). Skin lesions and arthritis initially got worse after starting treatment, which improved with addition of high dose steroid, (Prednisolone $60 \mathrm{mg}$ daily). Polyarthritis resolved five days after starting treatment with multidrug anti-leprosy medication and high
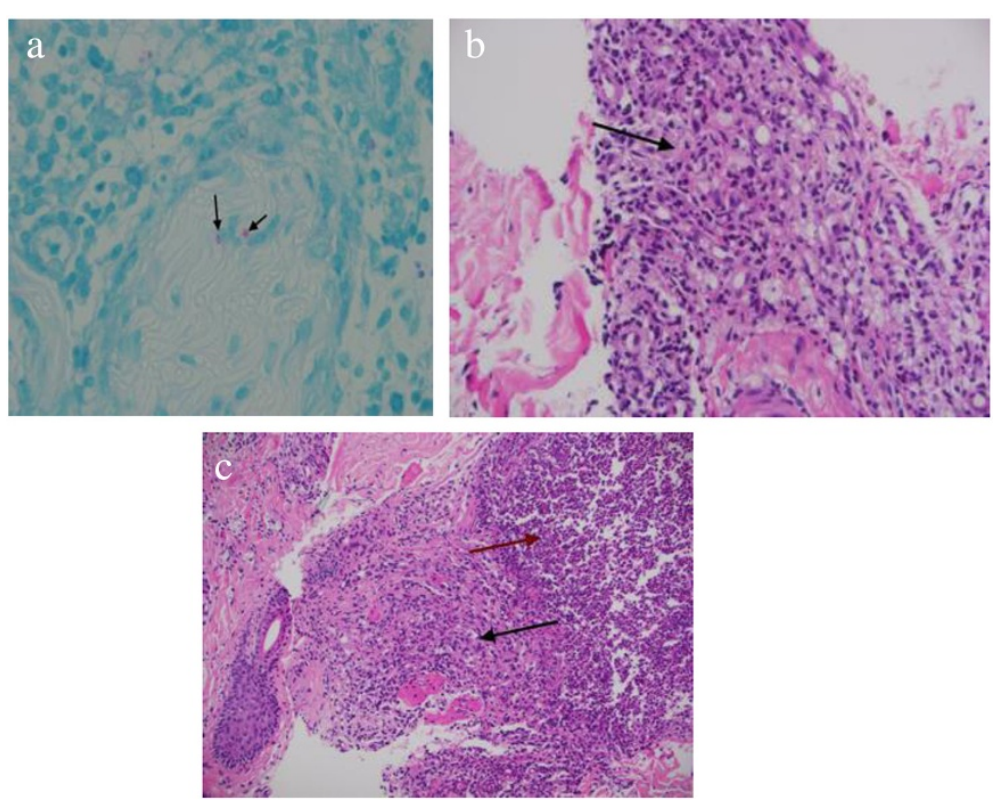

Figure 3 Histopathology of skin nodule. (a) Oil immersion wade fite stain showing acid-fast bacilli (arrows), (b) H\&E stain x 400 magnification showing granuloma (black arrow), (c) H\&E stain × 200 magnification showing granuloma (black arrow) and acute folliculitis with neutrophillic infiltrates (red arrow). 
dose steroid. Patient renal function returned to normal. Patient didn't undergo renal biopsy after improvement in his renal function. Patient was discharged in good general condition.

Patient had regular follow up in the out patient clinic with infectious disease doctor. Steroid was tapered slowly and patient was improving clinically. Four months after initiation of treatment, patient suddenly developed similar acute presentation. Patient was receiving monthly rifampicin $600 \mathrm{mg}$ and daily dapsone of $100 \mathrm{mg}$. Patient landed in emergency department with sudden onset of high grade fever, new skin painful nodular lesions, symmetrical polyarthritis involving small joints of hands, bilateral elbow joint, bilateral knees, ankles and bilateral metatarsophalangeal joints.

Laboratory investigations revealed leukocytosis, high inflammatory markers, and raised liver enzymes.

This episode was considered to be secondary to type 2-lepra reaction and treated accordingly. Patient responded well to $20 \mathrm{mg}$ of daily prednisolone. Patient is regularly followed up in infectious disease outpatient clinic and is receiving combined antileprosy medication for paucibacillary leprosy.

\section{Discussion}

Leprosy is a disease, which is more common in endemics areas and developing countries. Because of increase travel in today's world, it is possible that we may come across with leprosy patient in non-endemic areas. It is important to be updated about different manifestation of leprosy in order to avoid unnecessary diagnostic tests and to start proper and early treatment.

Exact pathogenesis of joint involvement in leprosy is still not fully elucidated. Lepra reactions (Graham et al. 2010; Bhat and Prakash 2012; Wakhlu and Agarwal 2010) (Types I and II lepra reaction), and direct infiltration (Wakhlu and Agarwal 2010) of the synovium by mycobacterium leprea are thought to be the underlying pathogenesis mechanisms for joint involvement. Peripheral sensory neuropathy (Wakhlu and Agarwal 2010) can also lead to Charcot's neuropathy.

There are two types of immunologic reactions to mycobacterium leprae antigens. These immunologic reactions can occur before, during or after the treatment of leprosy.

Type 1 Lepra (Graham et al. 2010; Bhat and Prakash 2012) reaction is a delayed hypersensitivity reaction. It is cell mediated immune response to $M$. leprae antigenic determinants and is characterized by acute inflammation of pre-existing skin lesions or by the appearance of new lesions and/or neuritis. Systemic involvement does not occur in type 1 reation.

Type 2 lepra (Graham et al. 2010; Bhat and Prakash 2012) reaction also termed as Erythema nodosum leprosum (ENL) is an immune complex mediated (type 3 hypersensitivity) response to M.leprae antigenic determinants. It results in severe painful skin lesion, nerve damage, fever and systemic manifestation. Systemic involvement can lead to arthritis, dactyltis, orchtitis, uveitis, lymphadenitis, glomerulonephritis, protienuria and hepatitis. It occurs mostly during the fist year of leprosy treatment. Recurrence of type 2 reaction is also common during treatment.

Chauhan et al (Wakhlu and Agarwal 2010) classify the arthritis in leprosy into the following groups: (1) Charcot's arthropathy secondary to peripheral sensory neuropathy; (2) swollen hands and feet syndrome; (3) acute polyarthritis of lepra reaction; and (4) chronic arthritis from direct infiltration of the synovium by lepra bacilli.

Shiva PRASAD (Prasad et al. 2012) and his team conducted retrospective case series study of leprosy patients. Forty-four cases with leprosy were identified. Musculoskeletal manifestations included arthritis $(n=22)$, swollen hands and feet syndrome $(n=11)$, tenosynovitis $(n=9)$, painful swollen feet $(n=9)$, arthralgia $(n=7)$ and vasculitis $(\mathrm{n}=1)$. Arthritis and tenosynovitis were part of spontaneous onset lepra reaction in 28 cases.

S L Atkin et al. (Atkin et al. 1990) conducted a study in seventy-seven patients with leprosy. Study showed that ten patients had generalized enthesitis and twenty patients had leprosy with manifestations of arthritis.

Another study, conducted by S L Atkin (Atkin et al. 1987) in patients with leprosy in Papua New Guinea, showed that 31 patients out of 55 had peripheral symmetrical inflammatory arthritis.

Hence it is important to include leprosy in list of possible differential diagnosis of arthritis, mainly in those countries where leprosy is prevalent or those patients who travelled from endemic areas.

Our patient's presentation with peculiar skin lesion combined with acute polyarthritis leads to diagnosis of leprosy.

Our patient presented with an acute onset of symmetrical polyarthritis and high-grade fever. High inflammatory markers, leukocytosis (Legendre et al. 2012) negative blood and urine cultures are supporting evidence for type 2 lepra reaction. Acute arthritis as well as enthesitis and tenosynovitis improved within a week after initiation of treatment.

Sometime, it is difficult to differentiate symmetrical polyarthritis due to leprosy from rheumatoid arthritis as in our patient. This question was entertained upon initial presentation of our patient with symmetrical polyarthritis. Absence of rheumatoid nodule, extra articular manifestation, patient being male, good response to anti leprosy treatment, and absence of Rheumatoid factor and anti-ccp are the clinical distinguishing features from rheumatoid arthritis in our patient.

At presentation patient also had acute renal impairment, which improved with hydration. Wide variety of renal involvement (Ahsan et al. 1995; Aggarwal et al. 2004) 
occurs in leprosy. Glomerular and interstitial lesions both can happen in leprosy. Glomerulonephritis, acute interstitial nephritis, tubulointerstitial nephritis, acute tubular necrosis and amyloidosis are important renal manifestation of leprosy.

Clinical feature of glomerulonephritis due to leprosy consist of asymptomatic hematuria and proteinuria. In some cases patient can also present with nephritic clinical picture. Immune complex mediated glomerulonephritis is the most common form of glomerulonephritis. ENL type 2 lepra (Ahsan et al. 1995; Aggarwal et al. 2004; Singhal et al. 1977) reaction is immune complex type 3 hypersensitivity reaction which can lead to immune complex mediated glomerulonephritis in leprosy.

Our patient had mild acute renal impairment with transient hematuria and proteinuria. In the presence of systemic manifestation, transient renal impairment was most likely secondary to type 2 lepra reaction.

The other interesting part in our case was second presentation, which was acute in onset. In spite on multidrug treatment and low dose steroid, patient developed this acute presentation. Patient developed new skin lesions, fever and polyarthritis. Laboratory investigation showed leukocytosis and raised liver enzyme. Question was raised whether patient has recurrence of leprosy disease or simply a lepra reaction. Possibility of drug induced liver injury was also entertained. It is interesting to note that recurrence (Graham et al. 2010; Bhat and Prakash 2012) of type 2 lepra can happen while patient on treatment. Type 2-lepra reaction can present with all these systemic manifestation including raised liver enzymes. Patient was managed as having type 2 lepra reaction and responded well.

\section{Conclusion}

Musculoskeletal manifestations are common in leprosy.

Leprosy should be included in differential diagnosis in those patients who present with typical skin rash and musculoskeletal manifestation. Type 2 lepra reaction, ENL, can be recurrent and usually presents with systemic manifestation involving skin, nerves, joint, kidney and liver as it happened in our patient.

Diagnosis of leprosy is easy but need to be thought off especially in migratory population to non-endemic areas.

\section{Competing interests}

The authors declare that they have no competing interests.

\section{Authors' contributions}

FA and SA wrote case report of "Case of arthritis secondary to leprosy". Both authors review the manuscript and approved it.

\section{Author details}

${ }^{1}$ Hamad Medical Corporation, P.O. Box 3050, Doha, Qatar. ${ }^{2}$ Hamad Medical Corporation, WCMC-Q, P.O. Box 3050, Doha, Qatar. ${ }^{3}$ Affiliated with Weill Cornell Medical College, Doha, Qatar.
Received: 18 November 2014 Accepted: 20 November 2014 Published: 15 December 2014

\section{References}

Aggarwal HK, Sharma P, Jaswal TS, Jain VK, Nand N, Sehgal R, Makkar V, Bharti K (2004) Evaluation of renal profile in patients of leprosy. JIACM 5(4):316-321

Ahsan N, Wheeler DE, Palmer BF (1995) Leprosy-associated renal disease: case report and review of the literature. J Am Soc Nephrol 5:1546-1552

Atkin SL, Welbury RR, Stanfield E, Beavis D, Iwais B, Dick WC (1987) Clinical and laboratory studies of inflammatory polyarthritis in patients with leprosy in Papua New Guinea. Ann Rheum Dis 46:688-690

Atkin SL, El-Ghobarey A, Kamel M, Owen JP, Dick WC (1990) Clinical and laboratory studies in patients with leprosy and enthesitis. Ann Rheum Dis 49:715-717

Bhat RM, Prakash C (2012) Leprosy: an overview of pathophysiology. Interdiscipl Perspect Infect Dis, Volume 2012, Article ID 181089, 6 pages doi:10.1155/ 2012/181089

Graham A, Furlong S, Margoles LM, Owusu K, Franco-Paredes C (2010) Clinical management of leprosy reactions. Infect Dis Clin Pract 18:235-238

Legendre DP, Muzny CA, Edwin S (2012) Hansen's disease (leprosy): current and future. Pharmacotherapy and treatment of disease-related immunologic reactions. Pharmacotherapy 32(1):27-37

Lopéz B, Mestre T, Grima B, Panarra A, Riso N (2012) Leprosy and rheumatoid arthritis: consequence or association? Celia Coelho Henriques. BMJ Case Reports, doi: 10.1136/bcr.12.2011.5346

Pardillo FE, Fajardo TT, Abalos RM, Scollard D, Gelber RH (2007) Methods for the classification of leprosy for treatment purposes. Clin Infect Dis 44:1096-1099

Prasad S, Misra R, Aggarwal A, Lawrence A, Haroon N, Wakhlu A, Krishnani N, Agrawal V, Paliwal VK, Jha S, Agarwal V (2012) Leprosy revealed in a rheumatology clinic: a case series. Int J Rheum Dis 16(2):129-133, do:10.1111/j.1756-185X.2012.01819.x. Epub 2012 Aug 31

Shejpal AR, Manohar T, Deshpande PK (2014) Hansen's arthritis: an overlooked entity. Vidarbha J Intern Med 16:43-45

Singhal PC, Chugh KS, Kaur S, Malik AK (1977) Acute renal failure in leprosy. Int J Lepr Other Mycobact Dis 45(2):171-174

Wakhlu A, Agarwal V (2010) Arthritis in leprosy by Sandeep Chauhan. Rheumatology 49:2237-2242, doi:10.1093/rheumatology/keq264. Advance Access publication 19 August 2010

WHO (2011) Classification of leprosy

World health organization (2014) Fact sheet $N^{\circ} 101 .$, Updated January 2014

doi:10.1186/2193-1801-3-734

Cite this article as: Alam and Emadi: Case of arthritis secondary to leprosy. SpringerPlus 2014 3:734.

\section{Submit your manuscript to a SpringerOpen ${ }^{\circ}$ journal and benefit from:}

- Convenient online submission

- Rigorous peer review

- Immediate publication on acceptance

- Open access: articles freely available online

- High visibility within the field

- Retaining the copyright to your article

Submit your next manuscript at springeropen.com 